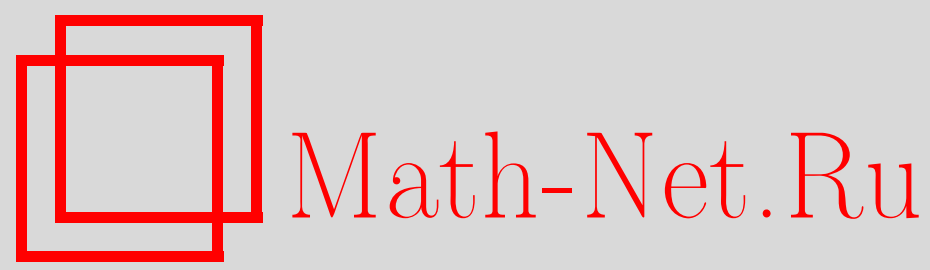

E. В. Щепин, Хаусдорфова размерность и динамика диффеоморфизмов, Матем. заметки, 1999, том 65, выпуск $3,457-463$

DOI: https://doi.org/10.4213/mzm1069

Использование Общероссийского математического портала Math-Net.Ru подразумевает, что вы прочитали и согласны с пользовательским соглашением http://www.mathnet.ru/rus/agreement

Параметры загрузки:

IP : 3.80 .253 .173

26 апреля 2023 г., 10:04:15

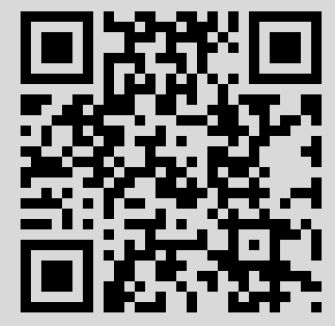




\title{
ХАУСДОРФОВА РАЗМЕРНОСТЬ \\ И ДИНАМИКА ДИФФЕОМОРФИЗМОВ
}

\section{Е. В. Щепин}

\begin{abstract}
В настоящей статье дано элементарное, не опирающееся на теорему Янга о когомологической размерности пространства орбит $p$-адического действия, доказательство гипотезы Гильберта-Смита для свободного липшицева действия. Получившийся результат оказался справедливым для класса пространств конечного хаусдорфова объема - класса существенно более шшрокого чем римановы многообразия. В качестве следствия из липшицевой гипотезы Гильберта-Смита получена теорема о том, что группа диффеоморфизмов конечномерного многообразия не имеет малых подгрупп.

Библиография: 12 названий.
\end{abstract}

1. Гипотеза Гильберта-Смита и динамика диффеоморфизмов. Гипотеза Гильберта-Смита утверждает, что всякая компактная группа, әффективно действующая на топологическом многообразии, является группой Ли. Под многообразием в этой работе мы понимаем многообразие $c$ краем. Гладкий вариант гипотезы Гильберта-Смита был доказан Бохнером и Монтгомери [1] еще в 1946 году. Альтернативные доказательства для гладкого случая содержатся в работах [2]-[4]. В частности, в работе Хана [2] содержится не только самое короткое доказательство гладкого случая, но там же имеется доказательство и для довольно широкого класса липшицевых действий. В полной общности гипотеза Гильберта-Смита для липшищевых действий доказана в [5]. Наконец, в [6] гипотеза Гильберта-Смита доказана для гёльдеровых действий с показателем гёльдеровости близким к единице.

Одним из ключевых понятий в решении пятой проблемы Гильберта [7] является понятие группы без малых подәрупn. Согласно теореме Петера-Вейля [7] всякая компактная группа, не являющаяся группой Ли, содержит бесконечную нульмерную компактную подгрупу. А компактная нульмерная группа имеет подгруппы, содержащиеся в любой окрестности единицы, т.е. является группой с малыми подгруппами. Таким образом, компактные групш Ли могут быть охарактеризованы как группы без малых подгрупп. Эта характеристика оказьвается справедливой и для локально компактных групп Ли [7]. Группу автодиффеоморфизмов гладкого многообразия $M^{n}$ обозначаем через Diff $M^{n}$ и рассматриваем ее с $C^{1}$-топологией, под которой подразумеваем топологию равномерной вместе с производными сходимости на компактах.

Одним из основных результатов этой заметки является следующая теорема.

Работа выполнена при поддержке Российского фонда фундаментальных исследований, грант № 96-01-01166a. 
ТеоремА 1. Для любого конечномерного гладкого многообразия $M^{n}$ группа его диффеоморфизмов Diff $M^{n}$ является группой без малых подгрупп.

Тот факт, что группа диффеоморфизмов не содержит мальх компактных подгрупп вытекает уже из теоремы Бохнера-Монтгомери [1] о том, что всякая локально-компактная группа, эффективно действующая на многообразии посредством диффеоморфизмов, является группй Ли. Но групша диффеоморфизмов не локально компактна, поэтому упомянутая выше теорема не следует из теоремы Бохнера-Монтгомери, а следует, как показано ниже, из липшицева варианта гипотезы Гильберта-Смита. Поскольку всякая группа содержит подгруппу порожденную одним элементом, вопрос об отсутствии малых подгрупп в групше диффеоморфизмов сводится к вопросу о динамике одного диффеоморфизма. И сформулированная вьше теорема 1 о группе диффеоморфизмов является следствием более точной теоремы о динамике диффеоморфизма, сформулированной ниже.

Как хорошо известно, гипотеза Гильберта-Смита равносильна следующей: әруппа иелых р-адических чисел $A_{p}$ не мохсет әффективно действовать на топологическом многообразии.

Доказательство эквивалентности классической и $p$-адической форм гипотезы Гильберта-Смита приведено в статье Эдвардса [8]. Изложим его вкратце.

В силу упомянутой выше теоремы Петера-Вейля достаточно доказать невозможность действия бесконечных компактных нульмерных групп на многообразиях. Но всякая компактная нульмерная группа либо содержит групш целых $p$-адических чисел $A_{p}$ либо содержит малые конечные подгрупшы. Второй вариант исключается в силу теоремы Ньюмена о периодических гомеоморфизмах.

Формулировке теоремы Ньюмена предпошлем несколько определений. Для любого автогомеоморфизма метрического пространства его орбитальным диаметром называем верхнюю грань диаметров его орбит, т.е. $\sup \operatorname{dist}\left(x, h^{n}(x)\right)$, где $x$ пробегает все точки пространства, а $n$ - все натуральные числа. Назовем периодическим орбитальным диаметром метрического пространства нижнюю грань орбитальньх диаметров его периодических гомеоморфизмов.

ТЕОРемА НЬЮменА. Периодический орбитальный диаметр топологического многообразия полохителен.

Теорема Ньюмена позволяет сформулировать гипотезу Гильберта-Смита в форме вопроса о динамике гомеоморфизма. Эта переформулировка была сделана Эдвардсом [8]: орбитальный диаметр динамически непрерывного гомеоморфизма топологического многообразия не уступает его периодическому орбитальному диаметру.

Здесь динамически непрерывным назьвается гомеоморфизм, совокупность итераций которого равностепенно непрерьвна. Докажем равносильность двух предложенных формулировок гипотезы Гильберта-Смита.

Всякий динамически непрерьвньй гомеоморфизм $h$ с конечным орбитальным диаметром порождает компактную подгруппу в групе всех гомеоморфизмов (замыкание множества его итераций) $G_{h}$ в силу теоремы Арцела. Тогда $G_{h}$ в силу оригинальной формулировки гипотезы Гильберта-Смита является групой Ли. А всякая группа Ли содержит элемент конечного порядка $g$. Но тогда орбитальньй диаметр $g$ не превосходит орбитального диаметра $G_{h}$, которьй совпадает с орбитальньм диаметром $h$, ведь 
любая орбита $G_{h}$ является замыканием некоторой орбиты $h$. Таким образом, форма Эдвардса вытекает из классической.

Обратно, если компактная групша $G$, действующая на многообразии, не является группой Ли, то, как было отмечено вьше, мы можем предполагать ее группой $A_{p}$. Переходя, если нужно, к подгруппе, мы можем добиться того, чтобы орбитальньй диаметр действия был меньше периодического орбитального диаметра многообразия. Но тогда гомеоморфизм, представленный любьм из элементов группы, динамически непрерьвен и имеет орбитальньй диаметр меньший ньюменовского вопреки форме Эдвардса.

Диффеоморфизм $h \in$ Auth $M$ называется динамически ограниченным по производной, если ограничена последовательность норм производных итераций как его самого, так и обратного к нему.

Теорема 1 является непосредственным следствием более точной теоремы 2. (Эта теорема по существу является ослабленной версией теоремы 1 из [9]. Усиленньй еевариант, при котором от диффеоморфизма требуется лишш поточечной динамической ограниченности производных, также верен, но его доказательство в этой заметке не приводится.)

ТЕорема 2 (о динамике диффеоморфизма). Орбитальный диаметр динамически ограниченного по производной диффеоморфизма не уступает орбитальному периодическому диаметру многообразия.

Риманова метрика на связном многообразии порождает обычную метрику, если расстояние между точками определить как длину минимальной геодезической их соединяющей. Так определенную метрику называют геодезической. Рассматриваемые римановы многообразия считаются наделенными именно этой метрикой.

Автогомеоморфизм метрического пространства называется динамически липииие$\boldsymbol{s} \boldsymbol{b}$, если все итерации его и обратного к нему гомеоморфизмов липшицевы с одной и той же константой $L$.

Очевидно, что всякий динамически ограниченньй по производной диффеоморфизм риманова многообразия является динамически липшицевым относительно соответствующей геодезической метрики.

Поэтому теорема 2 является следствием теоремы 3.

ТЕорема 3 (о динамике липпеоморфизма). Орбитальный диаметр динамически липиицева гомеоморфизма не уступает орбитальному периодическому диаметру риманова многообразия.

ЛЕмма (о компактном замыкании). Всякий динамически липиииев гомеоморфизм метрического пространства $M$ содержится в абелевой компактной липиичевой nodzpynne zpynnbl Auth $M$

ДокАЗАТЕЛЬСтво. Совокупность итераций динамически липшицева гомеоморфизма $h$ образует абелеву подгрупу $\left\{h^{n}\right\}-\infty<n<+\infty$ в $\operatorname{Auth}(M)$, имеющую компактное замькание. Действительно, это замыкание состоит из липшицевых гомеоморфизмов с константой Липшица $\lambda(h)=\sup \left\{\lambda\left(h^{n}\right) \mid n \in \mathbb{Z}\right\}$, и компактность его вытекает из теоремы Арцела.

ДоКАЗАТЕЛЬСТво ТЕОРЕМЫ 3. Пусть $h$ - динамически липшицев гомеоморфизм многообразия $M$. Обозначим через $G$ порожденную им компактную групп в Auth $M$. Тогда $G$ эффективно действует на $M$ лишшицевыми гомеоморфизмами и является согласно [5] группой Ли. Но во всякой орбите $G$ всюду плотна орбита липпеоморфизма $h$; 
поэтому орбитальньй диаметр действия $G$ совпадает с орбитальным диаметром $h$. Но всякая бесконечная группа Ли содержит окружность [7], а вместе с ней элемент конечного порядка. Орбитальный диаметр этого элемента, не уступая периодическому орбитальному диаметру многообразия, в то же время не превосходит орбитального диаметра $G$, откуда и вытекает нужное нам неравенство.

2. Изометрии пространств конечного объема. Теорема о динамике диффеоморфизма вьводится из гипотезы Гильберта-Смита для эффективных липшицевых действий. Предложенное автором в работе [5] доказательство гипотезы Гильберта-Смита для липшицевых действий опирается на теорему Янга [10] о том, что гомологическая размерность пространства орбит р-адического действия на п-мерном многообразии равна $n+2$. Таким образом, доказательство элементарной по формулировке теоремы о динамике диффеоморфизма оказьвается весьма неэлементарным.

В этом пункте дано элементарное доказательство гипотезы Гильберта-Смита, для липшицевых действий с бесконечными орбитами (в частности, для свободных действий) на любых пространствах локально конечного облема - класса пространств существенно более широкого, чем римановы многообразия.

Константой Липшица отображения $f: X \rightarrow Y$ метрических пространств назьвается точная верхняя грань отношений dist $f\left(x_{1}, x_{2}\right) / \operatorname{dist}\left(x_{1}, x_{2}\right)$, где $x_{1}, x_{2} \in X$, и обозначается через $\lambda(f)$. Липшицев гомеоморфизм, обратный к которому также липшищев, назьвается липпеоморфизмом или квазиизометрией.

Группу автогомеоморфизмов метрического пространства $M$ с топологией равномерной сходимости, порожденной $C^{0}$-нормой, обозначаем через $\operatorname{Auth}(M)$. Подгруппу в Auth $M$ называем липиииевой, если все ее элементы являются липшицевьги гомеоморфизмами. Поскольку групша вместе со всяким элементом содержит обратньй, все элементы липшицевой подгруппы являются липпеоморфизмами.

ЛЕмма (об общей константе) [5]. Пусть $G$ - компактная липиицева подгруппа в $\operatorname{Auth}(M)$. Тогда $\sup \{\lambda(g) \mid g \in G\}<\infty$.

Две метрики $\rho$ и $\rho^{\prime}$ на одном пространстве $M$ назьваются квазиизометричными, если тождественное отображение из $M, \rho$ в $M, \rho^{\prime}$ является квазиизометрией. Следующая лемма показьвает, что пространство с липшицевым действием компактной группы квазиизометрично пространству с изометричным действием.

ЛЕмма (об инвариантной метрике). Пусть $M, \rho$ - метрическое пространство, на котором липшицево действует компактная группа $G$. Тогда существует метрика $\rho^{\prime}$ на $M$, инвариантная по отношению $к$ действию $G$ и квазиизометричная $\rho$.

ДокАЗАТЕЛЬСТВо. Искомая метрика определяется следующей формулой

$$
\rho^{\prime}(x, y)=\int_{G} \rho(g(x), g(y)) d g
$$

Здесь интегрирование производится по мере Хаара на $G$. Подробности можно найти в [5].

Основным инструментом в данной статье являются понятия хаусдорфовой меры и размерности метрического пространства. Через $\mu_{t}(X)$ обозначается $t$-мерная мера Хаусдорфа множества $X$. Определения этих понятий можно найти в книге [11]. 
Хаусдорфовым облемом подмножества метрического пространства $M$ топологической размерности $k$ мы называем его $k$-мерную хаусдорфову меру.

Основным результатом этого пункта является

ТЕОРема 4 (о конечной орбите). Если нульмерная компактная группа липиицево действует на пространстве, содержащем непустое открытое мнохество конечного хаусдорфова обтема, то это действие имеет конечную орбиту.

Основной результат теории хаусдорфовой размерности теперь может быть сформулирован так.

ПРИНЦИП ПОЛОЖИТЕЛЬНОСТИ ОБЪЕМА. Хаусдорфов оббем любого непустого метрического пространства положителен (возможно бесконечен).

Следуюший факт просто следует из этого принципа и определений.

СЛЕДСТВИЕ. Хаусдорфова размерность пространства, имеющего конечный хаусдорфов обвем, совпадает с топологической.

Нам потребуется в дальнейшем следующие две леммы, первая из которых непосредственно вытекает из определения хаусдорфовой меры.

ЛЕмма (об увеличении меры). Липшичево $c$ константой $\lambda$ отображение $f$ : $X \rightarrow Y$ увеличивает $t$-мерную хаусдорфову меру $\mu_{\alpha}$ не более чем в $\lambda^{t}$ раз (т.е. $\left.\mu_{t}(f(X)) \leqslant \lambda^{t} \mu_{t}(X)\right)$.

ЛЕмма (об уменьшении меры). Пусть открытое нерастягивающее отображение $f: X \rightarrow Y$ в каждой точке имеет кратность $\geqslant n$. Тогда $n \mu Y \leqslant \mu X$ для любой хаусдорфовой меры.

ДокАЗАтЕЛЬСтво. Рассмотрим произвольную точку $y \in Y$. Фиксируем $n$ ее различных прообразов $x_{1}, \ldots, x_{n}$. Выберем у них непересекающиеся окрестности $O\left(x_{i}\right)$. Тогда в силу открытости $f$ окрестностью точки $y$ будет $O(y)=\bigcap_{i \leqslant n} f\left(O\left(x_{i}\right)\right)$. Положим $O_{i}=O\left(x_{i}\right) \cap f^{-1} O(y)$. Тогда $f\left(O_{i}\right)=O(y)$ при любом $i$, и множества $O_{i}$ дизъюнктны. Следовательно, для любого подмножества $S \subset O y$ будет $n \mu S \leqslant \mu\left(f^{-1} S\right)$, ибо прообраз $S$ содержит объединение дизъюнктных множеств $f^{-1}(S) \cap O_{i}$, каждое из которых в силу предыдущей леммы имеет меру большую либо равную мере $\mu S$. Далее из покрытия пространства $Y$ окрестностями типа $O(y)$ выберем счетное подпокрытие $\left\{O\left(y_{k}\right)\right\}$ и рассмотрим дизъюнктное покрытие $O_{k}=O\left(y_{k}\right) \backslash \bigcup_{i<k} O\left(y_{i}\right)$. Тогда $n \mu Y=n \sum \mu O_{k} \leqslant \sum \mu\left(f^{-1}\left(O_{k}\right)\right) \leqslant \mu X$, что и требовалось доказать.

Основным техническим результатом этой секции является следующая теорема (она уточняет теорему 2 из [8]).

ТЕОРема 5. Пусть компактная группа $G$ действует на метрическом пространстве конечного обгема $X$ посредством липиичевы $х$ гомеоморфизмов $и X_{\infty}$ является обвединением бесконечных орбит этого действия. Тогда $\operatorname{dim} X_{\infty} / G<\operatorname{dim} X$.

ДокАЗАТЕЛЬСтво. Заменим метрику на $X$ на инвариантную и квазиизометричную исходной в силу леммы об инвариантной метрике. Тогда липшицевость действия сохранится так же, как конечность объема $X$. А на пространстве орбит инвариантная метрика индуцирует метрику, по отношению к которой орбитная проекция является нерастягивающим отображением. 
Предположим, что напротив размерность $\operatorname{dim} X_{\infty} / G=n$ совпадает с размерностью $\operatorname{dim} X$. Тогда в силу принципа положительности объема $\mu_{n} X_{\infty} / G>0$. А так как объем $X_{\infty}$ не превосходит объема $X$, то он также конечен и найдется натуральное число $N$ такое, что вьполнено неравенство $\mu_{n}\left(X_{\infty}\right) \leqslant N \mu_{n} X_{\infty} / G$. Но кратность ограничения орбитальной проекции на $X_{\infty}$ больше чем $N$, поэтому в силу леммы об уменьшении меры имеем неравенство противоречащее предыдущему: $\mu_{n}\left(X_{\infty}\right) \geqslant N \mu_{n} X_{\infty} / G$.

Сразу отметим, что из доказанного утверждения вытекает такое следствие.

ТЕорема 6. Компактная группа, свободно и липиичево действующая на метрическом пространстве конечного хаусдорфова обгема, является группой Ли.

ДокАЗАТЕЛЬСТво. Поскольку всякая компактная группа, не являющаяся групой Ли, содержит бесконечную компактную нульмерную группу, предположив противное, мы получим, что существует метрическое пространство $M^{n}$ конечного объема и топологической размерности $n$ и бесконечная нульмерная компактная группа $G$ такие, что $G$ свободно и липшицево действует на этом пространстве. В этом случае все орбиты действия бесконечны и согласно теореме 5 размерность пространства орбит $\operatorname{dim} M^{n} / G$ должна быть меньше, чем $n$.

Но здесь мы вступаем в противоречие с классической формулой Гуревича для понижающих размерность отображений [12]. Из этой формулы вытекает, что отображение, прообразы точек при котором нульмерны (а именно, таким является орбитная проекция из $M^{n}$ на $\left.M^{n} / G\right)$, не может понижать размерность.

ДоКАЗАТЕЛЬСТВо ТЕОРЕмЫ 4. Пусть $\pi: X \rightarrow X / G$ орбитная проекция, группа $G$ нульмерна и компактна, а $U \subset X$ имеет конечный хаусдорфов объем. Фиксируем точку $p \in U$ все окрестности которой имеют размерность равную размерности $\operatorname{dim} U$. Тогда найдется такое $G^{\prime}$, являющееся открыто-замкнутой подгруппой $G$, что $G^{\prime}(p) \subset U$. Рассмотрим $U^{\prime}=\left\{x \in U \mid G^{\prime}(x) \subset U\right\}$. Тогда $U^{\prime}$ открыто, имеет ту же размерность, что $U$, и не больший хаусдорфов объем. Поэтому на основании теоремы 5 заключаем, что либо конечна $G^{\prime}(x)$ для некоторого $x \in U^{\prime}$, а тогда конечна и $G(x)$, ибо конечна факторгруппа $G / G^{\prime}$ и тогда наша теорема доказана, либо все орбиты действия $G^{\prime}$ на $U^{\prime}$ бесконечны, и поэтому $\operatorname{dim} U^{\prime} / G^{\prime}<\operatorname{dim} U^{\prime}$, что опять-таки противоречит тому, что нульмерные отображения не могут повышать размерность.

Поскольку в инвариантной метрике всякое действие липшицево и, более того, изометрично, гипотеза Гильберта-Смита для компактного случая может быть сформулирована следуюшим образом: группа изометрий компактного многообразия является әруппой Лu.

В этой формулировке ничего не говорится о метрике на многообразии, что означает ее произвольность. Эту произвольность можно несколько уменьшить, сведя вопрос только к метрикам индуцированньм из гильбертова пространства. А именно, если компактная группа $G$ действует на многообразии $M$, то на $M$ введем метрику $\rho$, индуцированную вложением в евклидово пространство. Тогда инвариантная метрика

$$
\rho^{\prime}(x, y)=\sqrt{\int_{G} \rho^{2}(g(x), g(y)) d g}
$$

будет иметь гильбертово происхождение. 
Соображение, что хаусдорфова размерность пространства орбит при изометричном действии не может быть меньше, чем хаусдорфова размерность исходного пространства, в сочетании с теоремой Янга [10] о том, что размерность пространства орбит на 2 больше размерности исходного многообразия в случае $p$-адического действия позволяют сформулировать следующий результат.

ТЕОРема 7. Если хаусдорфова размерность компактного многообразия меньие чем на 2 превосходит его топологическую размерность, то группа изометрий этого многообразия является группой Ли.

\section{СПИСОК ЦИТИРОВАННОЙ ЛИТЕРАТУРЫ}

[1] Bochner S., Montgomery D. Locally compact groups of differentiable transformations // Ann. of Math. (2). 1946. V. 47. P. 639-653.

[2] Hahn F.H. On the action of locally compact group of $E_{n} / /$ Pacific J. Math. 1961. V. 11. P. 221-223.

[3] Repovš D., Skopenkov A. B., Ščepin E. V. $C^{1}$-homogeneous compacta in $\mathbb{R}^{n}$ are $C^{1}$-submanifolds of $\mathbb{R}^{n}$ // Proc. Amer. Math. Soc. 1996. V. 124. № 4. P. 1219-1226.

[4] Repovš D., Skopenkov A. B., Ščepin E. V. Group actions on manifolds and smooth ambient homogeneity // J. Math. Sci. Dynam. Systems. 1997. V. 83. № 4. P. 546-549.

[5] Repovš D., Ščepin E. V. A proof of the Hilbert-Smith conjecture for actions by Lipschitz maps // Math. Ann. 1997. V. 308. P. 361-364.

[6] Малешич Й. Гипотеза Гильберта-Смита для гёльдеровых действий // УМН. 1997. Т. 52. № 2. C. $173-174$.

[7] Montgomery D., Zippin L. Topological Transformation Groups. Intersci. Tracts Pure Appl. Math. V. 1. Interscience Publ.: New York, 1955.

[8] Edwards R.D. Some remarks on the Hilbert-Smith conjecture // Proc. of the 4th Annual Western Workshop in Geometric Topology. Oregon: Oregon State Univ., 1987.

[9] Shchepin E. V. Hausdorff dimension and dynamics of diffeomorphisms // Topology and Applications. Intern. Topology Conference dedicated to P.S. Alexandroff's 100th Birthday (Moscow, May 27-31, 1996). Moscow: Phasis, 1996. P. 209-210.

[10] Yang C. T. p-adic transformation groups // Michigan Math. J. 1960. V. 7. P. 201-218.

[11] Hurewicz W., Wallman H. Dimension Theory. Princeton Univ. Press: Princeton, 1941.

[12] Engelking R. Dimension Theory. Warsaw.: PWN, 1978. 